\title{
Fatal catastrophic antiphospholipid syndrome at presentation of juvenile lupus
}

\author{
Debapoma Biswas', Saurabh Sutradhar ${ }^{2}$, Argha Rajbanshi², Priyankar Pal ${ }^{3}$ \\ From ${ }^{1}$ Resident, ${ }^{2}$ Senior Resident, ${ }^{3}$ Professor, Department of Pediatrics, Institute of Child Health, Kolkata, West Bengal, India
}

\begin{abstract}
Catastrophic antiphospholipid syndrome (CAPS) is a severe and rare form of antiphospholipid syndrome, extremely uncommon in the pediatric age group, characterized by multiple site thrombosis involving small, medium, and large blood vessels occurring over a short period of time (usually 1 week) causing multiorgan failure. We report the case of an 8-year-old girl presenting with fever, lymphadenopathy, and pain abdomen with refractory mitral regurgitation diagnosed as systemic lupus erythematosus with CAPS. All three antiphospholipid antibodies positivity in high titers further confirmed the diagnosis. In spite of the early initiation of adequate triple therapy (anticoagulation, steroids, and cyclophosphamide), our patient succumbed. The report aims to incorporate a greater awareness among clinicians for timely diagnosis and treatment of this condition and throws light on the varying ways in which lupus can present in children.
\end{abstract}

Key words: Anti-cardiolipin antibody, Antiphospholipid syndrome, Catastrophic antiphospholipid antibody, Juvenile lupus, Lupus anticoagulant

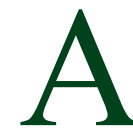

ntiphospholipid antibody syndrome (APS) is a heterogeneous multisystem autoimmune disorder of hypercoagulation and thromboembolic events in the presence of elevated antiphospholipid antibodies (aPL) [1]. The predominant antibodies associated are lupus anticoagulant, anticardiolipin, and beta 2 glycoprotein 1 . In primary APS, it may occur as an isolated entity and secondary APS; it is associated with other diseases such as systemic lupus erythematosus (SLE). Although there is no reliable data on the incidence of APS in children, reportedly $<3 \%$ of all APS occurs in childhood [2]. Since it is associated with high morbidity and mortality, the best approach seems to be early diagnosis and an aggressive treatment regimen.

Here, we present a case of probable catastrophic antiphospholipid syndrome (CAPS) with skin involvement and rapidly progressing encephalopathy, renal failure, and refractory cardiac failure.

\section{CASE REPORT}

An 8-year-old girl presented with complaints of high-grade intermittent fever for the past 1 month. There were associated pain abdomen, weight loss of $1.5 \mathrm{~kg}$, and neck nodes for the same duration. On examination, the child looked pale was tachycardic (heart rate $160 / \mathrm{min})$, hypotensive $(90 / 64 \mathrm{mmHg})$ with a systolic

\section{Access this article online}

Received - 28 September 2021

Initial Review - 21 October 2021

Accepted - 06 November 2021

DOI: $10.32677 /$ ijcr.v7i11.3119 murmur, and saturation in room air of $98 \%$. There were tender hepatomegaly and generalized lymphadenopathy.

Laboratory investigations showed anemia with a hemoglobin of $6.2 \mathrm{~g} / \mathrm{dl}$, positive direct Coombs' test, neutrophilic leukocytosis (total leukocyte count 16200/cumm, neutrophil 82\%), with hypoalbuminemia $(2.8 \mathrm{gm} / \mathrm{dl})$, C-reactive protein $3.2 \mathrm{mg} / \mathrm{L}$, and erythrocyte sedimentation rate of $68 \mathrm{~mm}$ in the $1^{\text {st }} \mathrm{h}$. Blood and urine cultures as well as screening for tuberculosis were negative. Lymph node biopsy revealed reactive follicular hyperplasia.

Within 5 days of admission, she started worsening; pain abdomen increased, with S3 gallop, and signs of cardiac failure. Simultaneously, there was the development of acute renal failure (urea $234 \mathrm{mg} / \mathrm{dl}$, creatinine $2.8 \mathrm{mg} / \mathrm{dl}$, with fractional excretion of sodium $>1$ ) along with hypoalbuminemia ( $2.3 \mathrm{~g} / \mathrm{dl}$ ), bicytopenia (platelet count 20,000/ cumm and hemoglobin of $5.9 \mathrm{~g} / \mathrm{dl}$ ), and deranged coagulation profiles (International Normalized Ratio 2.32). Echocardiography showed mitral leaflet prolapse with chordae tendineae tear, severe mitral regurgitation, and flow reversal in pulmonary veins. Antinuclear antibody was positive in 1:1000 titer (homogeneous), C3 $66 \mathrm{mg} / \mathrm{dl}$, $\mathrm{C} 4<6 \mathrm{mg} / \mathrm{dl}$, anti-ds DNA, and anti-Sm were negative.

She was diagnosed as SLE with CAPS and started on pulse methylprednisolone (30 $\mathrm{mg} / \mathrm{kg} /$ day), hydroxychloroquine with cardiotropic (milrinone), and inotropic support. B-type natriuretic peptide was $24,000 \mathrm{pg} / \mathrm{ml}$, immunoglobulin (Ig)M anti-beta2 glycoprotein1 (>200 RU/ml), IgM anti-cardiolipin antibody (118.19 $\mathrm{MPL} / \mathrm{ml}$ ), and lupus anticoagulant were all positive in high titers. She started having mucosal bleeds, erythroderma-like rashes with

Correspondence to: Dr. Priyankar Pal, Department of Pediatrics, Institute of Child Health, Kolkata, West Bengal, India. E-mail: mailme.priyankar@gmail.com

(C) 2021 Creative Commons Attribution-NonCommercial 4.0 International License (CC BY-NC-ND 4.0). 


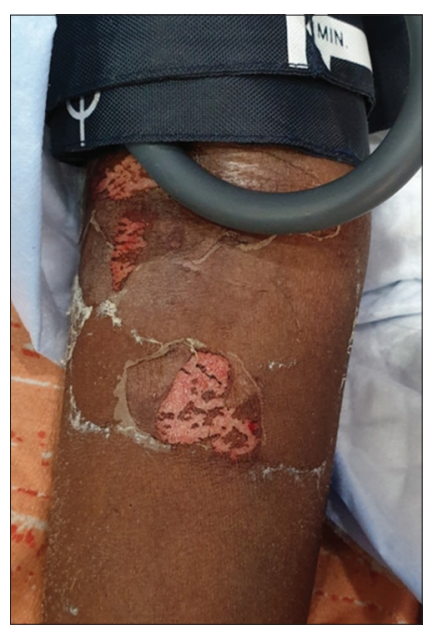

Figure 1: Necrosis of skin associated with microvascular thrombosis of dermal vessels

rapidly progressing epidermal necrosis, and peeling (Fig. 1). Because of the mucosal bleeds, unfractionated heparin was avoided and low molecular weight heparin (LMWH) was started but sensorium worsened with the development of severe encephalopathy. Following three methylprednisolone pulses, she was given one dose of intravenous (IV) cyclophosphamide $500 \mathrm{mg} / \mathrm{m}^{2}$, methylprednisolone pulses were continued for 2 more days. Cardiac failure continued to worsen and she was put on mechanical ventilation. However, the child suffered a cardiac arrest after 5 days and succumbed.

\section{DISCUSSION}

CAPS was first described by Asherson et al. in 1992, as a fatal variant of APS occurring in 1\% of APS patients [3], pediatric cases being rarer. The international pediatric APS registry revealed a mean age of onset of 10.7 years with a slight female preponderance [4]. Many children who are diagnosed to have primary APS on follow-up develop either SLE or an SLE-like illness.

As per the CAPS registry, the major organs involved during catastrophic episodes were renal (71\%), lungs (64\%), brain $(62 \%)$, heart $(51 \%)$, and skin $(50 \%)$, with variable affection of liver and gastrointestinal tract [5].CAPS associated with lupus is seen more in the younger population, shows more cerebral and cardiac involvement, and usually has a grave prognosis.

The diagnosis and treatment are challenging for physicians [6]. Due to its rapid and fatal course, management needs to be prompt. In 220 patients reported from the CAPS registry, more than half had an infection as a precipitating factor and $4 \%$ had lupus flares [7]. Anti-cardiolipin antibodies are detected in $84 \%$ of the patients, the lupus anticoagulant in $76 \%$, the antinuclear antibodies in $62 \%$, antidouble-stranded DNA in 36\%, and anti-Ribonucleoprotein in $8 \%$ of CAPS patients [8]. Thrombocytopenia is found in $63-68 \%$ of the patients and hemolytic anemia in 26-32\%. Cardiac symptoms include valve abnormalities (thickening and vegetations), coronary artery disease, myocardial dysfunction, pulmonary hypertension, and intracardiac thrombi. In a meta-analysis of aPL-associated heart valvular diseases (HVD) in SLE patients, nearly 90\% had positive aPL compared to $44 \%$ without HVD [9].
All these findings correlated with our case as well. Although microthrombosis is one of the typical histological markers of a CAPS event, it may be difficult to confirm and the diagnosis in our patient could only be labeled as "probable" CAPS. The extensive skin necrosis (Fig. 1) that was clinically evident possibly resulted from cutaneous microvascular thrombosis, but considering the moribund condition of the child a skin biopsy was not done.

A combination of steroids, anticoagulants, and drugs reducing aPL titers such as IVIg or plasmapheresis form the backbone of the treatment protocol. The addition of cyclophosphamide decreases mortality in SLE-CAPS as per the international registry $[10,11]$. Our patient was initiated on methylprednisolone pulses and LMWH for the CAPS. However, she developed multiorgan failure within $48 \mathrm{~h}$, and administration of cyclophosphamide too was unable to halt the progression and allow administration of other therapies such as rituximab or plasmapheresis.

\section{CONCLUSION}

An extremely rare occurrence in children, the case is a prototype of CAPS that can be rapidly fatal even with early institution of therapy.

\section{REFERENCES}

1. Lim W. Antiphospholipid syndrome. Hematology Am Soc Hematol Educ Program 2013;2013:675-80.

2. Madison JA, Zuo Y, Knight JS. Pediatric antiphospholipid syndrome. Eur J Rheumatol 2020;7 Suppl 1:1-10.

3. Asherson RA, Cervera R, de Groot PG, Erkan D, Boffa MC, Piette JC, et al. Catastrophic antiphospholipid syndrome: International consensus statement on classification criteria and treatment guidelines. Lupus 2003;12:530-4.

4. Avcin T, Cimaz R, Rozman B. The Ped-APS registry: The antiphospholipid syndrome in childhood. Lupus 2009;18:894-9.

5. Cervera R, Bucciarelli S, Plasín MA, Gómez-Puerta JA, Plaza J, PonsEstel G, et al. Catastrophic antiphospholipid syndrome (CAPS): Descriptive analysis of a series of 280 patients from the "CAPS registry". J Autoimmun 2009;32:240-5.

6. Erkan D, Espinosa G, Cervera R. Catastrophic antiphospholipid syndrome: Updated diagnostic algorithms. Autoimmun Rev 2010;10:74-9.

7. Cervera R Front J, Gomez-Puerta JA, Espinosa G, Cucho M, Bucciarelli S, et al. Validation of the preliminary criteria for the classification of catastrophic antiphospholipid syndrome. Ann Rheum Dis 2000;64:1205-9.

8. Asherson RA, Cervera R, Piette JC, Font J, Lie JT, Burcoglu A, et al. Catastrophic antiphospholipid syndrome. Clinical and laboratory features of 50 patients. Medicine (Baltimore) 1998;77:195-207.

9. Hojnik M, George J, Ziporen L, Shoenfeld Y. Heart valve involvement (Libman-Sacks endocarditis) in the antiphospholipid syndrome. Circulation 1996;93:1579-87.

10. Bayraktar UD, Erkan D, Bucciarelli S, Espinosa G, Asherson R, Catastrophic Antiphospholipid Syndrome Project Group. The clinical spectrum of catastrophic antiphospholipid syndrome in the absence and presence of lupus. J Rheumatol 2007;34:346-52.

11. Ortel TL, Erkan D, Kitchens CS. How I treat catastrophic thrombotic syndromes. Blood 2015;126:1285-93.

Funding: None; Conflicts of Interest: None Stated.

How to cite this article: Biswas D, Sutradhar S, Rajbanshi A, Pal P. Fatal catastrophic antiphospholipid syndrome at presentation of juvenile lupus. Indian J Case Reports. 2021;7(11):489-490. 